\title{
Resistance to Ditylenchus destructor Infection in Sweet Potato by the Expression of Small Interfering RNAs Targeting unc-15, a Movement-Related Gene
}

\author{
Weijuan Fan, Zhaorong Wei, Min Zhang, Peiyong Ma, Guiling Liu, Jianli Zheng, Xiaoding Guo, and Peng Zhang
}

First, second, third, and eighth authors: National Key Laboratory of Plant Molecular Genetics, Institute of Plant Physiology and Ecology, Shanghai Institutes for Biological Sciences, Chinese Academy of Sciences, 300 Fenglin Road, Shanghai 200032, China; fourth and seventh authors: Institute of Food Crops, Jiangsu Academy of Agricultural Sciences, 50 Zhongling Street, Nanjing 210014, China; fifth and sixth authors: Tai'an Academy of Agricultural Science, 16 Tailai Road, Tai'an, Shandong 271000, China; and eighth author: Shanghai Key Laboratory of Plant Functional Genomics and Resources, Shanghai Chenshan Plant Science Research Center, Chinese Academy of Sciences, Shanghai Chenshan Botanical Garden, 3888 Chenhua Road, Shanghai 201602, China.

Accepted for publication 27 May 2015.

\begin{abstract}
Fan, W., Wei, Z., Zhang, M., Ma, P., Liu, G., Zheng, J., Guo, X., and Zhang, P. 2015. Resistance to Ditylenchus destructor infection in sweet potato by the expression of small interfering RNAs targeting unc-15, a movement-related gene. Phytopathology 105:1458-1465.

Stem nematode (Ditylenchus destructor) is one of most serious diseases that limit the productivity and quality of sweet potato (Ipomoea batatas), a root crop with worldwide importance for food security and nutrition improvement. Hence, there is a global demand for developing sweet potato varieties that are resistant to the disease. In this study, we have investigated the interference of stem nematode infectivity by the expression of small interfering RNAs (siRNAs) in transgenic sweet potato

that are homologous to the unc-15 gene, which affects the muscle protein paramyosin of the pathogen. The production of double-stranded RNAs and siRNAs in transgenic lines with a single transgene integration event was verified by Northern blot analysis. The expression of unc-15 was reduced dramatically in stem nematodes collected from the inoculated storage roots of transgenic plants, and the infection areas of their storage roots were dramatically smaller than that of wild-type (WT). Compared with the WT, the transgenic plants showed increased yield in the stem nematode-infested field. Our results demonstrate that the expression of siRNAs targeting the unc-15 gene of $D$. destructor is an effective approach in improving stem nematode resistance in sweet potato, in adjunct with the global integrated pest management programs.
\end{abstract}

Plant-parasitic nematodes are major agricultural pathogens that cause severe damage and huge agricultural losses annually on a global scale (Atkinson et al. 2012; Jones et al. 2013; Nicol et al. 2011; Oerke 2006). Ditylenchus destructor Thorne, commonly known as stem nematode, is one of the major pathogens of sweet potato (Ipomoea batatas) and causes significant yield loss of 20 to $50 \%$; in endemic regions, there is a $100 \%$ yield loss (Xie et al. 2004; $\mathrm{Xu}$ et al. 2015; Zhang et al. 2006). In China, sweet potato is the fourth largest crop, and the parasitic nematode causes severe losses in the major growing regions, especially in North and East China (Xu et al. 2015). Because the situation is worsening with each passing year, an effective technique for the management of the disease is necessary (Xie et al. 2004; Xu et al. 2015). Currently, nematicides, crop rotation, and the use of resistant varieties are practical techniques for controlling plant-parasitic nematode diseases (Overstreet 2009). However, nematicides cause environmental pollution and have a deleterious effect on human health; crop rotation has some intrinsic limitations; and the narrow genetic background of cultivated sweet potato for nematode resistance makes it difficult to breed resistant cultivars by traditional breeding (Xie et al. 2004). Genetic engineering represents a promising approach for generating novel germplasm that is resistant to the parasitic nematode, and might additionally lead to a sustainable and effective control of the disease (Atkinson et al. 2012).

Corresponding author: P. Zhang; E-mail address: zhangpeng@sibs.ac.cn

http://dx.doi.org/10.1094/PHYTO-04-15-0087-R

(c) 2015 The American Phytopathological Society
The main symptoms of stem nematode infection in sweet potato are stem necrosis and storage root rot; hence, severe infection often results in a dramatic decrease in yield or no yield (Scurrah et al. 2005). The above-ground part of infected sweet potato plants generally displays a weak disease phenotype, or none at all during the entire planting period, until the rot storage roots are finally harvested. Hence, the nature of the stem nematode infection also increases the difficulty of early diagnosis and control of the disease (Dutta et al. 2015; Wan et al. 2008). Study of stem nematode has been focusing on the analysis of population development, infestation development, and pathogenicity (Jones et al. 2013; Subbotin et al. 2005). Only recently, improved host resistance to stem nematode has been reported by the introduction of protein inhibitors (Gao et al. 2011) or molecular markers (Zhou et al. 2005), in sweet potato. In contrast to the stem nematode, most researches have been focused on cyst and root-knot nematodes for their broad host range and devastating impact on global agriculture (Charlton et al. 2010; Fairbairn et al. 2007; Fanelli et al. 2005; Huang et al. 2006; Yadav et al. 2006). Consequently, this knowledge has served as a useful guide in stem nematode studies.

RNAi technology has been useful for studying the interaction between plant host and parasitic nematode (Dutta et al. 2015). RNAi effects on Caenorhabditis elegans genes provided useful references and predictions for potential RNAi effects of homologous genes in plant-parasitic nematode (http://www.wormbase.org). For example, screening of candidate genes of Heterodera glycines, whose homologous genes in $C$. elegans have lethal phenotypes or phenocopies, revealed that the silencing of some genes, e.g., $H g$-rps-23, by feeding on the double-stranded RNAs (dsRNAs) of the target genes led to loss of viability in the worm or development 
of fewer females (Alkharouf et al. 2007; Klink et al. 2007). The expression of hair-pin dsRNAs of nematode target genes in the host plants, following the dsRNA degradation into small interference RNAs (siRNAs) that silence the target gene of the nematode, has been proved to be effective in improving host resistance to nematode (Charlton et al. 2010; Dutta et al. 2015; Yadav et al. 2006). Successful RNAi target genes include a large number of parasite genes that expressed in the esophageal gland cells of nematodes and related to specific functions of the nematode such as penetration, migration, and maintenance (Bakhetia et al. 2008; Davis et al. 2008; Dinh et al. 2014; Huang et al. 2006; Souza Ddos et al. 2011).

In addition to the parasitic genes in nematodes, research was also focused on host plant genes, such as those expressing cellulose binding protein, 1,4- $\beta$-glucanase gene family, and pathogenesis-related genes (Hamamouch et al. 2011; Hewezi et al. 2008; Wieczorek et al. 2008). Extensive genetic research on the host and parasite enabled a deeper insight into their interaction (Abad and Williamson 2010).

In order to effectively control stem nematode growth and propagation in sweet potato, it was opined that it would be suitable to interfere with the function of movement. Since movement is essential for its life cycle, interfering with muscle function might greatly affect nematode infectivity and propagation. The unc-15 gene codes for paramyosin, which is a major thick filament component, playing an important role in muscle assembly (Benian et al. 2006). In nematodes, most muscle cells are located in the body wall, which aids in locomotion. RNAi phenotypes of the unc-15 gene in C. elegans show severe symptoms, such as, being lethal to the larva, abnormal egg, abnormal locomotion, etc. (Fraser et al. 2000). This study investigated the interference effect of siRNAs homologous to the $u n c-15$ gene in transgenic sweet potato.

\section{MATERIALS AND METHODS}

Stem nematode cDNA library construction and sequence analysis. Stem nematodes (D. destructor) were collected from infected storage roots of cultivar Xushu22 after 30 days inoculation at room temperature. The cDNA library of stem nematode was constructed by Shanghai OE Biotech Co. Ltd. (Shanghai, China). Briefly, mRNAs were isolated from all stages of stem nematode reproduction and used to synthesize cDNAs by mRNA reverse transcription. These cDNA fragments were then inserted into a pMD$18 \mathrm{~T}$ vector (TakaRa Dalian Co. Ltd., China) and transformed into E.coli DH5 $\alpha$. About 2,000 clones were randomly selected and used for plasmid DNA extraction; sequencing was done using ABI 3730 DNA analyzer. Sequence analysis was performed using the blastx program in the blastall package (NCBI, version 2.2.9).

Identification of target genes and construction of RNAi binary vector. After sequencing, D. destructor unc-15 clones were identified from the cDNA library. A 350-bp fragment encoding 116 amino acid C-terminal UNC-15 (Fig. 1A) was selected as the target region for RNAi. The sense and antisense fragments of unc-15 were inserted into a modified cloning vector pBlueScript (PBS) that harbors a synthetic intron-containing hairpin expression cassette. The synthetic intron is an AU-rich intron (Syn7) that can be processed efficiently in plant cells (Goodall and Filipowicz 1991). The hair-pin dsRNA-expressing cassette from the PBS was subcloned into the binary vector pCAMBIA1301 to generate the final binary vector pC-RNAi-unc15 (Fig. 1B). Primers used for vector construction are listed in Table 1.

Genetic transformation of sweet potato and transgenic plant regeneration. Sweet potato cultivar Xushu22, a popular starch-rich variety in North and East China, was used for the production of transgenic plants. The Agrobacterium tumefaciens strain LBA4404 harboring pC-RNAi-unc15 was used for sweet potato transformation as described by Yang et al. (2011). Briefly, embryogenic suspension of Xushu22 was used as explant for cocultivation with the LBA4404 strain harboring pC-RNAi-unc15, and transformed suspension clusters were selected from the SBM medium
(MS plus $\mathrm{VB}_{1}$ at $0.3 \mathrm{mg} /$ liter plus sucrose at $30 \mathrm{~g} /$ liter) containing hygromycin at $10 \mathrm{mg} / \mathrm{liter}$, for $1 \mathrm{month}$. Then, hygromycin-resistant calli or somatic embryos were isolated and regenerated in the fresh SBM-cf200 medium (SBM plus cefotaxime at $200 \mathrm{mg} / \mathrm{liter}$ ) containing hygromycin at $10 \mathrm{mg} /$ liter. Regenerated plantlets were propagated and rooted on fresh regeneration medium. Independent transgenic plants were transferred to the greenhouse and fields for subsequent analysis.

Molecular analysis of transgenic plants by Southern and Northern blots. Total genomic DNA was isolated from the leaves of in vitro plants by the method described by Kim and Hamada (2005). DNA $(20 \mu \mathrm{g})$ was digested with HindIII, which is a unique restriction enzyme site in the T-DNA region of pC-RNAi-unc15 (Fig. 1B). A 500-bp DIG-labeled DNA fragment of $h p t$ was amplified using the following specific primers: $5^{\prime}$-TTCTACACAGC CATCGGTCC-3' and 5'-CCCATGTGTATCACTGGCAA-3' with PCR DIG Probe Synthesis Kit (Roche Applied Science, Mannheim, Germany). Gel electrophoresis, DNA blot, hybridization, and detection were conducted using Roche hybridization and detection kit according to manufacturer's instruction (Roche Applied Science).

Northern blot was performed by the method described by Huang et al. (2006) with minor modification. Small RNAs were extracted from the mature leaves of greenhouse plants by using the mirVana miRNA isolation kit (Ambion, Austin, TX). Aliquots of $15 \mu \mathrm{g}$ of RNAs were separated on $15 \%$ acrylamide, $7 \mathrm{M}$ urea, and 1×Trisborate-EDTA gels, and electro-transferred onto Hybond-XL nylon membranes (Amersham Biosciences, Piscataway, NJ) in $0.5 \times$ Trisborate-EDTA buffer. Probes of the target gene were labeled from the gel-purified restriction fragments of unc-15 with biotin-dUTP using the North\&South Biotin Random Prime DNA Labeling Kit (Thermo Scientific, Rockford), respectively. Hybridization was performed at $41^{\circ} \mathrm{C}$ for $16 \mathrm{~h}$ using North\&South Chemiluminescent Hybridization and Detection Kit (Thermo Scientific). Stringency wash was performed at room temperature, and the membranes were exposed to X-ray films for $2 \mathrm{~h}$ before taking photos.

Plant growth evaluation in greenhouse and fields. The shoots of transgenic plants in bottles were transferred to round plastic pots (diameter, $35 \mathrm{~cm}$; height, $25 \mathrm{~cm}$ ) containing a mixture of vermiculite, turf and humus $(1: 1: 1, \mathrm{vol} / \mathrm{vol} / \mathrm{vol})$ at a greenhouse in SIPPE, Shanghai for further growth. When transgenic plants grew for 2 to 3 months in pots, stems with apical part were cut $(20 \mathrm{~cm}$ length) from the plants for field propagation. For infection trial, wild-type (WT) and transgenic plants were transferred to the field at the same time in the experimental station of Jiangsu Academy of Agricultural Sciences, Nanjing, Jiangsu province. Thirty stems per line were randomly planted in the nursery field infested with stem nematodes ( $>100 / \mathrm{g}$ of soil). Simultaneously, five stems per line were planted in a normal field. Field conditions were the same in the nursery field and normal field. After 5 months of growth, the storage roots of WT and transgenic plants were harvested and their yields were recorded. Plant phenotype was recorded by photography during the growth period.

Storage root infection assessment by artificial inoculation. Storage roots harvested from normal field after 5 months of growth were used for assessing the extent of infection. Nematode $D$. destructor strain was provided by M.-S. Lin, Nanjing Agricultural University, and maintained by infected storage roots of sweet potato. Stem nematode was inoculated into storage roots of WT sweet potato for propagating controls. Second-stage juveniles (J2) of stem nematode were hatched from the egg mass collected from the infected sweet potato storage roots and used as inoculum (in 30\% glycerol) for the subsequent infection assay (Southey 1986). Nematode inoculation assay was performed using the method described by Lin (1989). Each welldeveloped and healthy storage root was inoculated with approximately $1,000 \mathrm{~J} 2$ nematodes and the inoculated storage roots were maintained in an incubator in the dark at $22^{\circ} \mathrm{C}$. Five storage roots per line were chosen for the nematode inoculation assay. All infection assays were conducted in triplicate. 
To evaluate resistance of nematode infection by sweet potato, infection areas of the inoculated storage roots from the WT and transgenic plants were recorded 45 days after infection as described with modifications (Xie et al. 2009; Xie et al. 2010). The severity of disease symptoms in mature storage roots was evaluated on a scale of 0 to 4 ( 0 , no symptom; 1 , faint rotten, less than $5 \%$ rotten; 2 , middle rotten, 5 to $10 \%$ rotten; 3 , severe rotten, up to $50 \%$ rotten; and 4 , severe rotten, with 50 to $100 \%$ rotten). The severity was evaluated using imageJ software (Schneider et al. 2012).

Gene expression analysis of nematodes cultivated by transgenic plants. Storage roots were infected by stem nematodes at different inoculation times and infected samples used for RNA preparation were harvested from the storage roots around the inoculation regions. For quantitative real-time reverse transcription polymerase chain reaction (qRT-PCR), total RNAs were extracted from the samples mixed by at least three storage roots per line using RNAplant Plus Reagent (Tiangen Biotech, Beijing, China) as per the protocol provided by the manufacturer. Aliquots $(2 \mu \mathrm{g})$ of total RNAs were reverse transcribed using $20 \mu \mathrm{l}$ of ReverTra Ace (Toyobo, Osaka, Japan). The resulting cDNA was subsequently diluted 10-fold with buffer. Gene-specific primers of the unc-15 gene were designed as shown in Table 1. In order to avoid the amplification of regions from RNAi construct in the host plant, the primers for qRT-PCR were designed to amplify the N-terminal sequence from 49 to $200 \mathrm{bp}$ of UNC-15. PCR $(50 \mu \mathrm{l})$ was performed in SYBR Green PCR Master Mix (Applied Biosystems, Foster City, CA), along with $1 \mu \mathrm{l}$ of cDNA template and $5 \mu \mathrm{M}$ each of forward and reverse primers. The PCR cycling parameters were set at $95^{\circ} \mathrm{C}$ for $10 \mathrm{~min}$, followed by 40 cycles for $95^{\circ} \mathrm{C}$ for $15 \mathrm{~s}$ and $60^{\circ} \mathrm{C}$ for $1 \mathrm{~min}$ in CFX96 thermocycler (Bio-Rad, CA). qRT-PCRs were performed in triplicate with WT as negative control. The $D$. destructor actin gene (GenBank accession no. JN248784) was used as an internal control. The relative fold change was calculated according to the $2^{-\Delta \Delta C T}$ method (Livak and Schmittgen 2001). All experiments were repeated three times, and the Student $t$ tests were performed for significance analysis.

\section{RESULTS}

Identification of unc-15 gene of $D$. destructor from a cDNA library. In $C$. elegans, muscles are characterized by

A

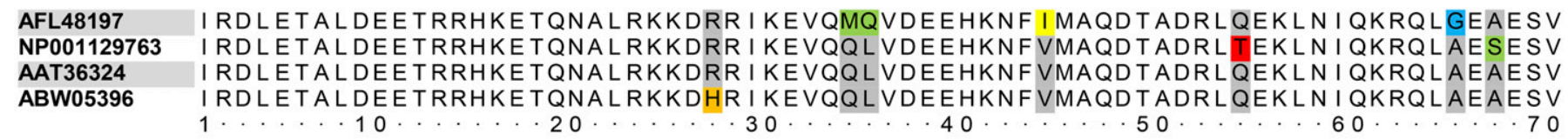
AFL48197 TMQNQRVRRYQRELEDAEGRADQAESSLHL IRAKHRSSVVTGKS NP001129763 TMQNQRVRRYQHELEDAEGRADQAESSLHL IRAKHRSSVVANK AAT36324 TMANQRVRRYQHELEDAEGRADQAESSLHL IRAKHRSSVVTGK ABW05396 TMANQRVRRYQHELEDAEGRADQAESSLHL IRAKHRSSVVTGKT $71 \cdots 80 \cdots 10 \ldots \ldots 110 . \cdots$

B

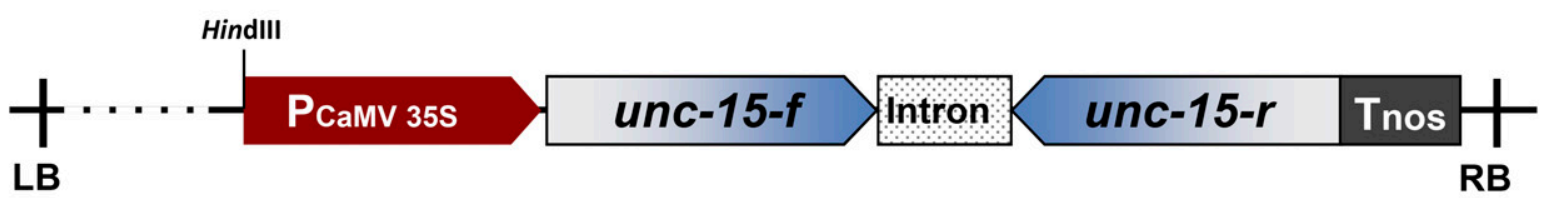

C

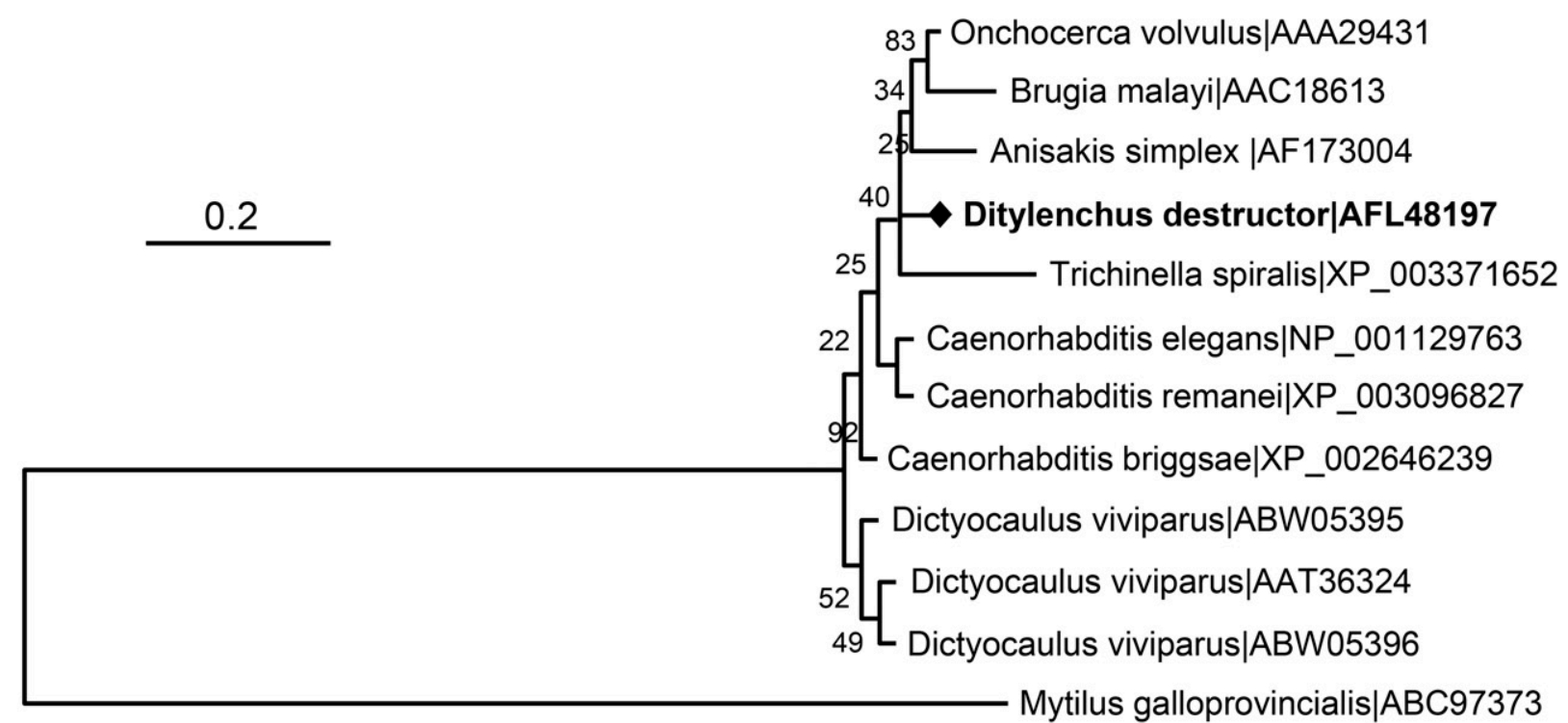

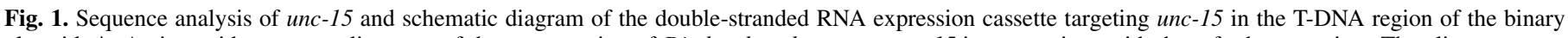

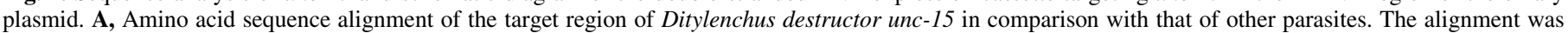

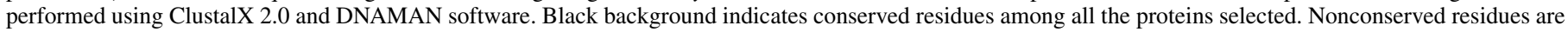

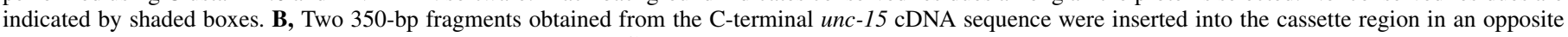

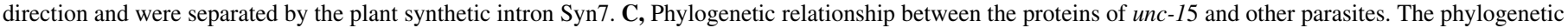
tree was generated by the neighbor-joining method using MEGA 5.0. 
a dense body and M-line. Thick filaments are composed of myosin (UNC-54), paramyosin (UNC-15), and myosin heavy chain A (MYO-3), and anchored to the M-line on one side and bound to the dense body on the other side by the protein titin (Klink et al. 2007). UNC-15 of nematodes is an important component of muscle constructs. Two thousand clones from a cDNA-library of $D$. destructor were randomly selected for sequencing, in order to identify the unc-15 gene. Several clones of the candidate unc-15 gene and their full cDNA length were assembled from derived sequences (GenBank accession no. JN216830.1). The phylogenetic tree analysis (Fig. 1C) of unc-15 shows that unc-15 in D. destructor has $91 \%$ identity with homologous genes in C. elegans, C. briggsae, and $C$. remanei, has $90 \%$ identity with that in Dictyocaulus viviparous (AAT36324), and 89\% with that in Dictyocaulus viviparous (ABW05396), and identity values with other nematode species were $\leq 88 \%$. This gene was used as the target gene for interfering D. destructor development and growth by RNA silencing. Since their C-terminal showed relatively less sequence homology among aligned sequences (Fig. 1A), the mRNA sequence of this region was selected as target for constructing RNAi expressing cassette (Fig. 1B).

Production and Southern blot analysis of unc-15 RNAi transgenic plants. The embryogenic suspensions of Xushu22 induced from shoot meristems were used for Agrobacteriummediated transformation of sweet potato (Yang et al. 2011). In total, 21 independent transgenic lines transformed with pC-RNAi-unc 15 were produced and propagated in vitro by shoot culturing. The success of T-DNA integration was verified by Southern blot analysis, wherein more than half of transgenic plant lines had undergone single insertion events (14 single copy lines). Of these, five unc-15 RNAi plant lines having a single integration event (Fig. 2A) were transferred into pots for further growth, and eventually planted into field for phenotype analysis.

DsRNA and siRNA detection in unc-15 RNAi transgenic plants. To confirm the proper expression of hair-pin dsRNA,
Northern analyses were performed using the small RNAs extracted from the leaves of greenhouse-grown transgenic plants. Two $\sim 20-$ to 24-nt siRNA bands and the 300-bp dsRNA fragment of unc-15 (Fig. 2B) were detectable in all the RNAi transgenic plants, after hybridization with biotin-labeled $u n c-15$ probe, indicating that the dsRNAs expressed from the RNAi cassette of transgenic plants could be successfully processed to form siRNAs. Among the five test lines, unc-15-4 detected the weakest signal of siRNA, although its dsRNA signal was the strongest.

Phenotype and yield evaluation of unc-15 RNAi transgenic plants. Under the normal greenhouse condition, all the plant lines showed a normal development and growth was indistinguishable between WT and transgenic plants (Fig. 3A). Once the plants were transferred to the field, all transgenic lines showed a healthy growth, and produced storage roots without any changes in yield, when compared with WT after 5 months (Fig. 3B). In order to study the effects of stem nematode infection on storage root production, the WT and transgenic plants were also grown in the nematodeinfested field for 5 months, simultaneously. After harvesting, four of five tested transgenic plants produced significantly more storage root biomass than WT plants in the nematode-infested field (Fig. 3C). The line unc-15-4 showed no difference with WT control, possibly due to its low siRNA level as shown in Figure 2B. These results imply that the transgenic plants developed a high resistance to stem nematode infection and performed better under the biotic stress.

Stem nematode infection assay of harvested storage roots by artificial inoculation. As sweet potato resistance assessment toward stem nematode in the field is time-consuming and unreliable (Gao et al. 2011), early screening of nematode resistance in sweet potato was unsuitable. In laboratory, artificial inoculation is often used as an alternative approach for sweet potato resistance evaluation. After inoculation, the brown infection region around the inoculation spot in sweet potato storage root demonstrated the extent of infection (Fig. 4). The nematodes usually develop

TABLE 1. Primers used for amplifying gene fragments of double-stranded RNA expression cassette and target and nontarget genes in polymerase chain reaction (PCR) analyses

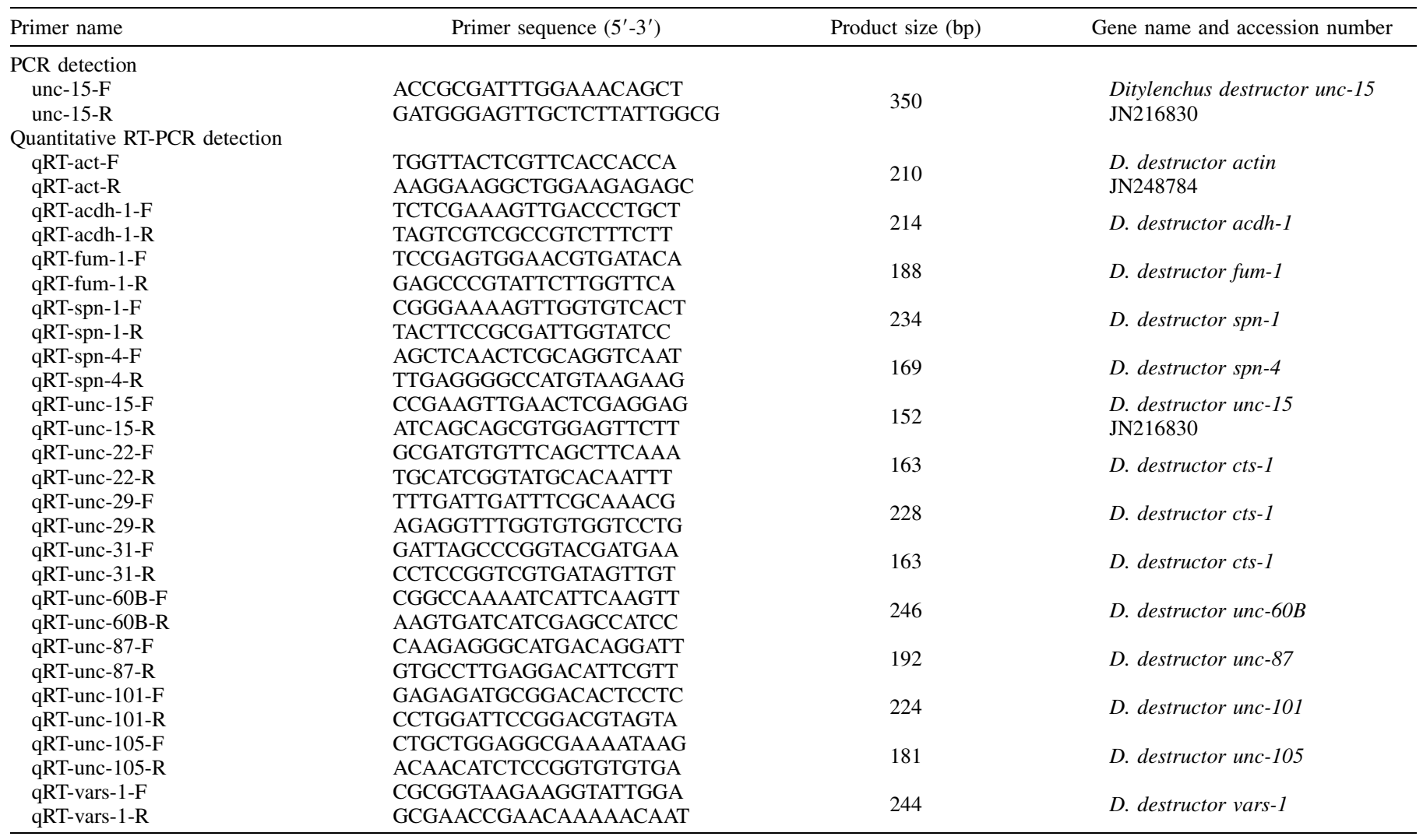


a concentric circle infection area that is seen in transverse sections of the sweet potato storage root from the inoculation site. The resistance evaluation method of Xie et al. $(2009,2010)$ was adopted, with some modifications. Infection areas observed in the transverse sections of storage root were evaluated 45 days postinfection (Fig. 4). The results showed a significant difference in storage roots between WT and transgenic plants. The WT storage roots showed the typical severe symptom of storage decay due to the infection by stem nematodes. Some roots showed completely rotted with white eggs of stem nematodes while others were partially infected (Fig. 4A). The infection area of transgenic storage root was considerably lower than that of WT storage roots. Compared with WT's, the infection areas in transgenic lines were lower by at least $50 \%$ (Fig. 4B). Some inoculated storage roots of transgenic lines showed no significant spreading of nematodes in the inoculation site (Fig. 4A). Weak rot around their outer layer was detected in some plants. These observations confirmed that the transgenic lines were able to develop resistance against stem nematode infestation. These results indicated that the RNAi of target gene unc-15 satisfactorily interrupted the nematode development, which led to less damage to sweet potato storage roots.

qRT-PCR analysis of genes related to stem nematode locomotion. To assess whether the expression of target gene unc15 in stem nematode was interfered, the mRNA transcript levels after inoculation of storage roots were performed by qRT-PCR. There was no significant change in the expression of unc-15 in stem nematodes that fed on WT plants, at all time points. This was
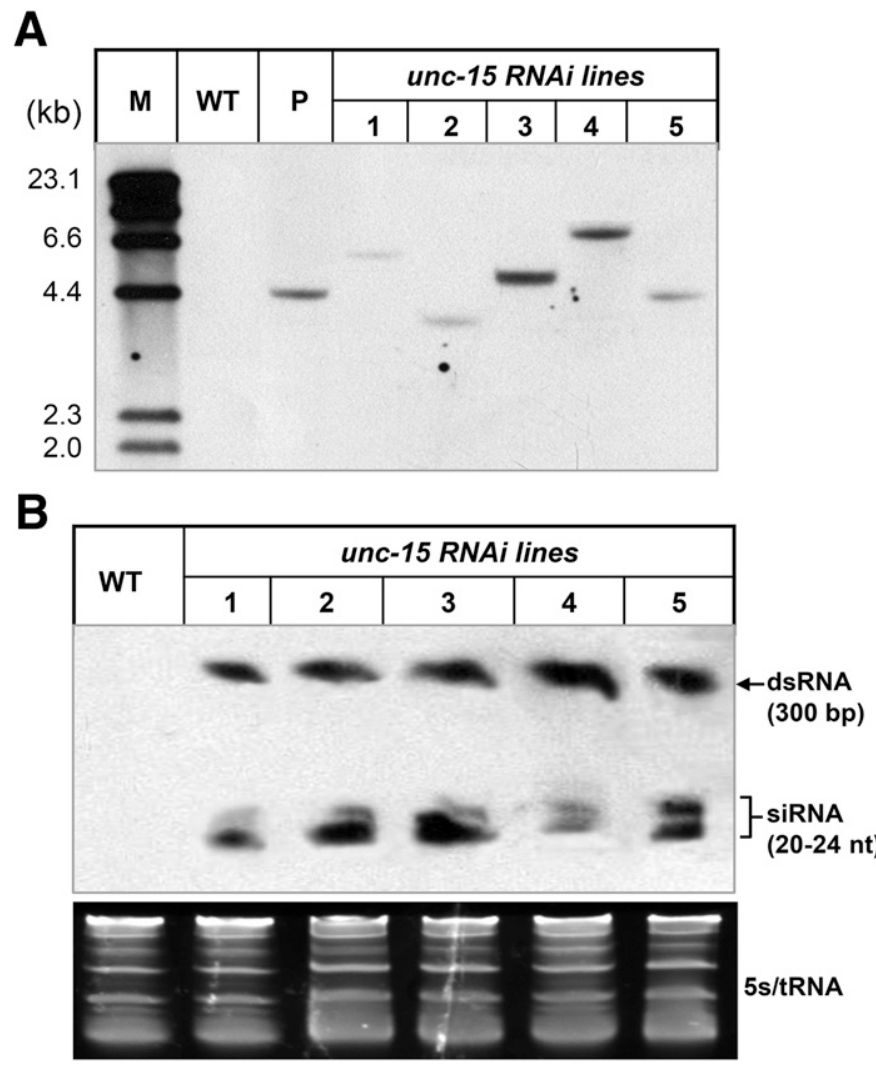

Fig. 2. Molecular characterization of $u n c-15$ RNAi transgenic sweet potato by Southern and Northern blot analyses. A, Integration pattern of T-DNA in the unc-15 RNAi plant lines detected by DIG-labeled hygromycin transferase gene (hpt) probe in Southern analysis. Genomic DNA $(20 \mu \mathrm{g})$ extracted from transgenic plants and wild-type (WT) plants were digested with HindIII. M, DNA molecular marker; P, plasmid control digested by HindIII; WT, wild-type plant of cultivar Xushu22; Line 1 to 5, different unc-15 RNAi transgenic plant lines. B, Double-stranded RNA and siRNA detection in unc-15 RNAi transgenic lines (1 to 5) with 300-bp biotin-labeled $u n c-15$ probe in Northern analysis. Small RNA $(15 \mu \mathrm{g})$ extracted from leaves of WT and unc-15 RNAi transgenic plants was used. consistent with the stem nematode life style, which migrates in sweet potato storage root throughout its life, with no sedentary stage. In contrast, mRNA transcript of unc-15 was significantly down regulated by two to three folds in the transgenic storage roots at all magnifications (Fig. 5). The results showed that the targets gene was silenced after stem nematode infection of transgenic storage roots.

A
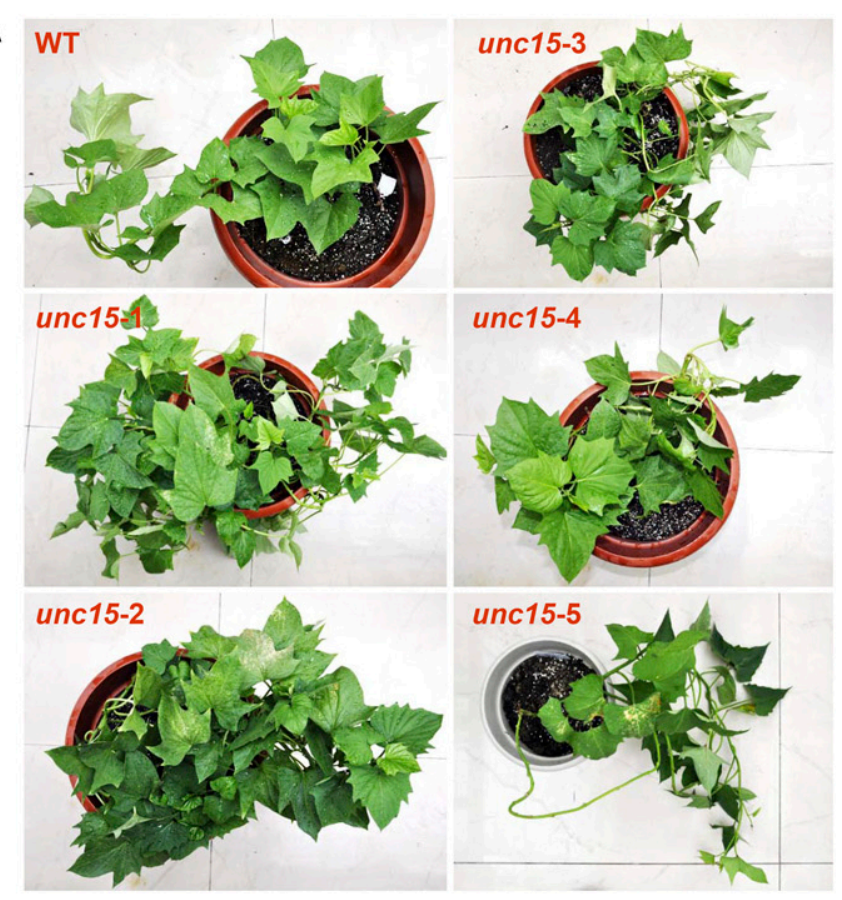

B
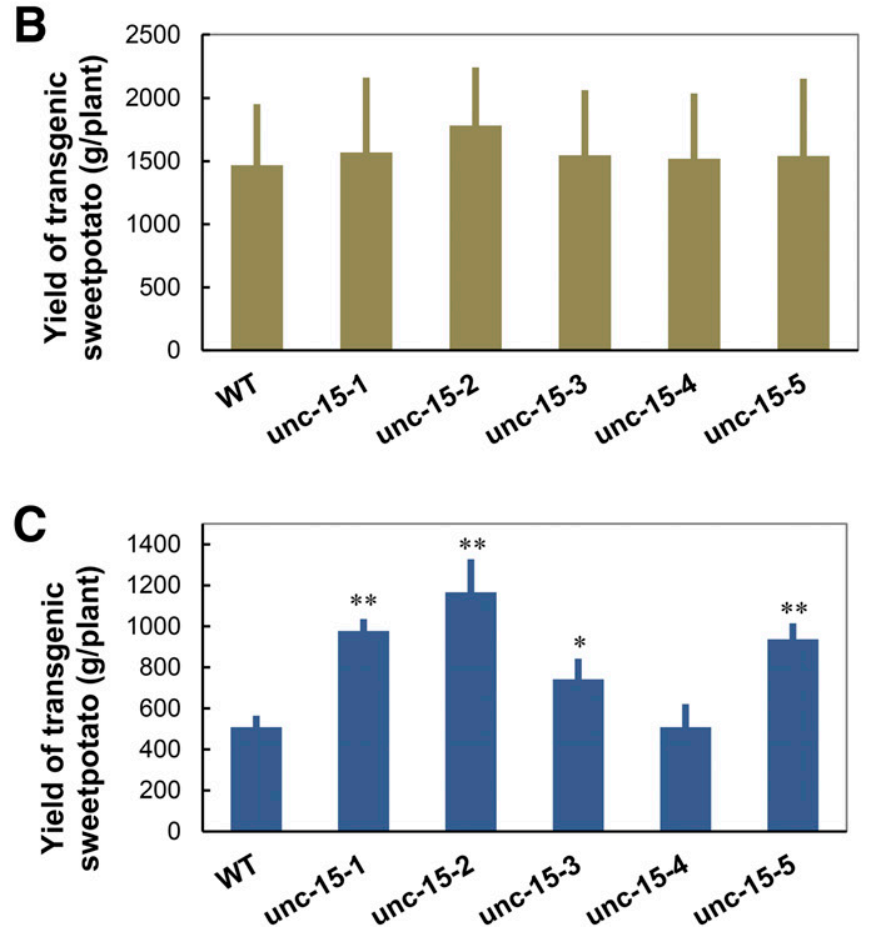

Fig. 3. Phenotypic evaluation of wild-type (WT) and unc-15 RNAi transgenic plant lines in greenhouse and field conditions under the normal and stem nematode-infested fields after 5-month growth. A, Plant growth status in pots under greenhouse condition. B, Storage root biomass of WT and the transgenic plants in a normal field. Each bar represents the mean + standard error (SE) of five plants. C, Storage root biomass of the transgenic and WT plants in stem nematode-infested field. Each bar represents the mean + SE of 30 plants. Asterisks indicate a significant difference from that of WT at $* P<0.05$ or ** $P<0.01$ by the Student's $t$ test. 
To further check the impact of silencing of $u n c-15$ on the expression of other genes that were related to movement, 12 genes were further analyzed for their expression in storage roots at 30 days postinfection. Three of the twelve genes, unc-105, unc-29, and unc-31, were found to have altered expression levels, especially unc-29 (Fig. 5B). These changes indicate that the silencing of unc-15 expression directly affect the function of the close-knit UNC family genes.

\section{DISCUSSION}

Improvement in resistance toward stem nematode infection has become an important objective in sweet potato breeding and field management. In this study, transgenic sweet potato plants that expressed siRNA targeting to $u n c-15$ were obtained. The results of this study reveal that transgenic plants have normal phenotype and high resistance toward stem nematode infection, both in field
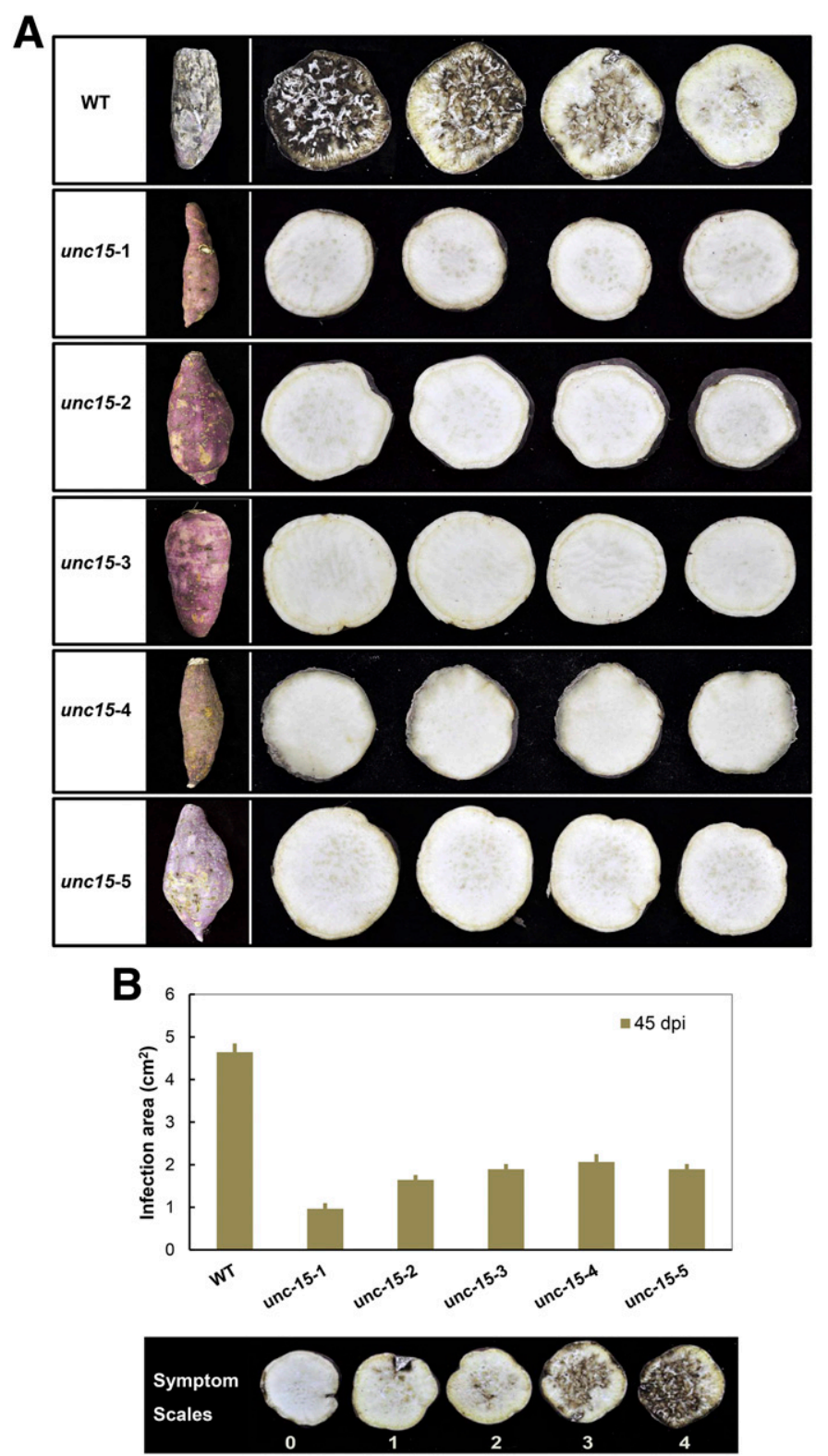

Fig. 4. Comparison of stem nematode resistance in storage roots of wild-type (WT) and unc-15 RNAi transgenic plants after 45 days post inoculation (dpi). A, Extent of rot in the storage roots. At least three storage roots per line were used for the assay. B, Evaluation of infected areas in storage roots according to the symptom scales. Each bar represents the mean + standard error (SE) of three storage roots per line. All transgenic lines showed significantly less infection than that of WT plants $(P<0.05$ and 0.01 , Student $t$ test $)$. evaluation and artificial inoculation assay, than that of WT plants. This indicates that interference in stem nematode movement by the expression of siRNAs targeting to $u n c-15$ in sweet potato is effective.

It is widely accepted that the expression of hair-pin dsRNA in plant hosts could have an interfering effect on its pathogen, in which the dsRNA of the host could be degraded to siRNAs for silencing the target gene. This technique has been explored successfully in cyst and root-knot nematodes (Charlton et al. 2010; Fairbairn et al. 2007; Fanelli et al. 2005; Huang et al. 2006; Ibrahim et al. 2011; Li et al. 2010; Urwin et al. 2002; Yadav et al. 2006). To select the target genes, one strategy was based on RNAi with evident phenotypes of corresponding genes in $C$. elegans. The lethal phenotype was preferred for selecting homologous genes of the pathogen. For example, Alkharouf et al. (2007) obtained many candidates genes of $H$. glycines that were homologous with $C$. elegans genes with a lethal phenotype and one of these genes, $\mathrm{Hg}$-rps-23, was silenced successfully leading to death of the nematode. The expression pattern of the movement-related genes that belong to the uncoordinated (unc) family was also investigated in $H$. glycines (Klink et al. 2007). The UNC family members perform many important functions in C. elegans, including focal adhesion, architecture, and stimulation, thus rendering a lethal phenotype by RNAi (Zengel and Epstein 1980). UNC-15 plays an important role in nematode movement, and an RNAi phenotype in the larva is lethal in C. elegans.
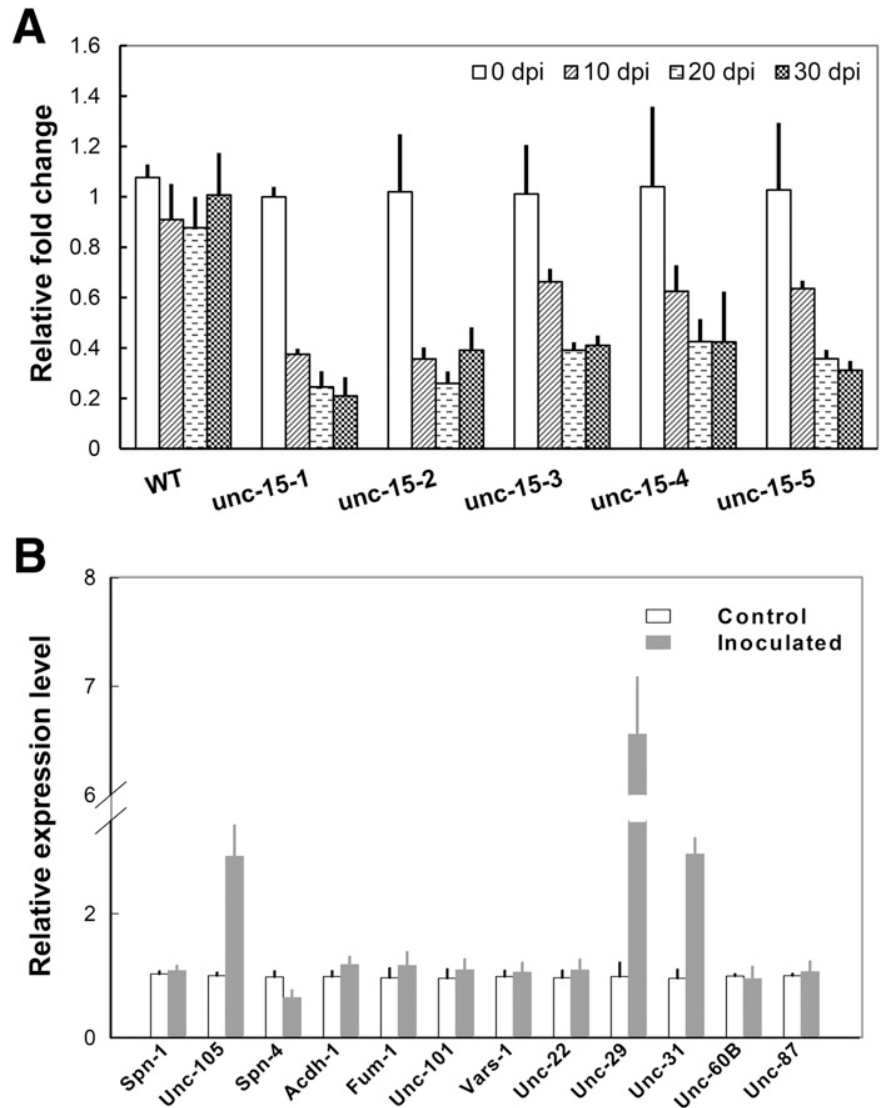

Fig. 5. Quantitative real-time reverse transcription polymerase chain reaction (qRT-PCR) analysis of $u n c-15$ and other 12 movement-related genes in stem nematodes collected from inoculated storage roots. A, Transcript changes of unc-15 gene in infected wild-type (WT) and five transgenic lines at 0, 10, 20, and 30 days postinfection (dpi). B, Transcript changes of 12 movement-related genes at 30 days postinfection in the unc-15-1 transgenic line. The sample at 0 dpi served as a control. spn-1, serpin 1; unc-105, degenerin; spn-4, SPiNdle orientation defective 4; acdh-1, acyl-CoA dehydrogenase 1; fum-1, fumarase 1; unc-101, adaptin; vars-1, valyl-tRNA synthetase; unc-22, twitchin; unc-29, nonalpha subunit of the nicotinic acetylcholine receptor ( $\mathrm{AChR}$ ) superfamily; $u n c-31$, pleckstrin; unc-60B, cofilin; and $u n c-87$, actin-bundling protein. Each bar represents the mean + standard error of qRT-PCR reactions performed in triplicate. 
In our study, interferences in the unc-15 expression in D. destructor by expressing siRNAs of unc-15 reduced its infectivity and rendered the transgenic plant resistant to stem nematode infection.

RNAi mechanism is well known in plant, but reports on simultaneous detection of dsRNA and siRNA in transgenic plants (Huang et al. 2006) were limited. In our study with transgenic sweet potato, both dsRNA and siRNA were detected by Northern blot analysis, indicating that the simultaneous detection of dsRNA by hair-pin dsRNA-expressing cassette and siRNAs in sweet potato cells was feasible. Transgenic sweet potato plants expressing with unc-15 siRNAs developed resistance to stem nematode in their storage roots, thus proving the feasibility of studying the interaction between plant host and pathogen. The results of our study confirmed a change in the expression of other native genes in stem nematodes collected from inoculated storage roots of transgenic plants.

Differences in the expression pattern of unc-15 in D. destructor and $H$. glycines in nontransgenic plants might be due to differences in their life cycle. As a major component of muscles, thick filaments are composed by myosin (UNC-54), paramyosin (UNC-15), and myosin heavy chain A (MYO-3) in C. elegans (Klink et al. 2007). The unc-15 is expressed at all stages of the lifecycle in C. elegans. This result was in agreement with $D$. destructor parasite life cycle that does not have sedentary stage and syncytium formation, and they are in constant locomotion in sweet potato storage root throughout their entire life cycle. In $H$. glycines, unc- 15 expression level was the lowest at 15 days postinfection and the highest at the $\mathrm{J} 2$ stage, which is suitable for its sedentary stage with less movement (Klink et al. 2007).

There are two different strategies in sweet potato to avoid the nematode infection namely, anti-invading and anti-extending. Proper assessment of the resistance is necessary. Currently, the two different approaches for evaluating sweet potato resistance toward stem nematodes is either by field evaluation in stem nematodeinfested field or by artificial inoculation of stem nematode in harvest storage roots (Xie et al. 2010). In a field, stem nematode can cause dramatic reduction in sweet potato yield, when their population is high; and because this duration lasts several months, it is the most reliable method for screening resistant cultivars. In our study, the yield of sweet potato in the infested field was significantly lower than the unc-15 RNAi transgenic plant lines, which had a better yield. This suggests that the transgenic plants were truly resistant to stem nematodes. The resistance capacity in transgenic plants was further confirmed by the artificial inoculation of mature storage roots. One major difference in artificial inoculation was the evasion of the epidermis barrier by the stem nematodes during invasion, thereby facilitating a direct entry of the nematodes into the inner tissues of sweet potato storage roots. Our results on artificial inoculation clearly demonstrate that the passage of stem nematodes inside the transgenic storage roots was repressed, as evidenced by a reduction in the rotten area around the inoculation sites.

In summary, the RNAi approach is an efficient tool for improving resistance to stem nematode by sweet potato or for studying the host-pathogen interaction. The siRNA generated in the storage roots of transgenic sweet potato silenced the unc-15 gene expression and changed the expression of other nontarget genes in stem nematode, thus rendering the transgenic plants resistant to stem nematode infections. This study showed that targeting the interference of stem nematode movement is feasible in sweet potato for achieving resistance against stem nematode.

\section{ACKNOWLEDGMENTS}

We thank M.-S. Lin of the Nanjing Agricultural University for providing the nematode $D$. destructor. This work was supported by the National High Technology Research and Development Program of China (No. 2012AA101204, 2011AA100607), the National Natural Science Foundation of China (31201254), the Science and Technology Commission of
Shanghai Municipality (14DZ 2260400), and Shanghai Municipal Afforestation \& City Appearance and Environmental Sanitation Administration (F132427, G152429).

\section{LITERATURE CITED}

Abad, P., and Williamson, V. M. 2010. Plant nematode interaction: A sophisticated dialogue. Adv. Bot. Res. 53:147-192.

Alkharouf, N. W., Klink, V. P., and Matthews, B. F. 2007. Identification of Heterodera glycines (soybean cyst nematode [SCN]) cDNA sequences with high identity to those of Caenorhabditis elegans having lethal mutant or RNAi phenotypes. Exp. Parasitol. 115:247-258.

Atkinson, H. J., Lilley, C. J., and Urwin, P. E. 2012. Strategies for transgenic nematode control in developed and developing world crops. Curr. Opin. Biotechnol. 23:251-256.

Bakhetia, M., Urwin, P. E., and Atkinson, H. J. 2008. Characterisation by RNAi of pioneer genes expressed in the dorsal pharyngeal gland cell of Heterodera glycines and the effects of combinatorial RNAi. Int. J. Parasitol. 38:1589-1597.

Benian, G. M., Mercer, K. B., Miller, R. K., Tinley, T. L., Sheth, S., and Qadota, H. 2006. Caenorhabditis elegans UNC-96 is a new component of M-lines that interacts with UNC-98 and paramyosin and is required in adult muscle for assembly and/or maintenance of thick filaments. Mol. Biol. Cell 17:3832-3847.

Charlton, W. L., Harel, H. Y., Bakhetia, M., Hibbard, J. K., Atkinson, H. J., and McPherson, M. J. 2010. Additive effects of plant expressed doublestranded RNAs on root-knot nematode development. Int. J. Parasitol. 40: 855-864.

Davis, E. L., Hussey, R. S., Mitchum, M. G., and Baum, T. J. 2008. Parasitism proteins in nematode-plant interactions. Curr. Opin. Plant Biol. 11:360-366.

Dinh, P. T., Zhang, L., Brown, C. R., and Elling, A. A. 2014. Plant-mediated RNA interference of effector gene Mc16D10L confers resistance against Meloidogyne chitwoodi in diverse genetic backgrounds of potato and reduces pathogenicity of nematode offspring. Nematology 16:669-682.

Dutta, T. K., Banakar, P., and Rao, U. 2015. The status of RNAi-based transgenic research in plant nematology. Front. Microbiol. 5:760.

Fairbairn, D. J., Cavallaro, A. S., Bernard, M., Mahalinga-Iyer, J., Graham, M. W., and Botella, J. R. 2007. Host-delivered RNAi: An effective strategy to silence genes in plant parasitic nematodes. Planta 226:1525-1533.

Fanelli, E., Di Vito, M., Jones, J. T., and De Giorgi, C. 2005. Analysis of chitin synthase function in a plant parasitic nematode, Meloidogyne artiellia, using RNAi. Gene 349:87-95.

Fraser, A. G., Kamath, R. S., Zipperlen, P., Martinez-Campos, M., Sohrmann, M., and Ahringer, J. 2000. Functional genomic analysis of C. elegans chromosome I by systematic RNA interference. Nature 408:325-330.

Gao, S., Yu, B., Yuan, L., Zhai, H., He, S. Z., and Liu, Q. C. 2011. Production of transgenic sweet potato plants resistant to stem nematodes using oryzacystatin-I gene. Sci. Hortic. (Amsterdam) 128:408-414.

Goodall, G. J., and Filipowicz, W. 1991. Different effects of intron nucleotide composition and secondary structure on pre-mRNA splicing in monocot and dicot plants. EMBO J. 10:2635-2644.

Hamamouch, N., Li, C., Seo, P. J., Park, C. M., and Davis, E. L. 2011. Expression of Arabidopsis pathogenesis-related genes during nematode infection. Mol. Plant Pathol. 12:355-364.

Hewezi, T., Howe, P., Maier, T. R., Hussey, R. S., Mitchum, M. G., Davis, E. L., and Baum, T. J. 2008. Cellulose binding protein from the parasitic nematode Heterodera schachtii interacts with Arabidopsis pectin methylesterase: Cooperative cell wall modification during parasitism. Plant Cell 20:3080-3093

Huang, G. Z., Allen, R., Davis, E. L., Baum, T. J., and Hussey, R. S. 2006. Engineering broad root-knot resistance in transgenic plants by RNAi silencing of a conserved and essential root-knot nematode parasitism gene. Proc. Natl. Acad. Sci. USA 103:14302-14306.

Ibrahim, H. M., Alkharouf, N. W., Meyer, S. L., Aly, M. A., Gamal El-Din Ael, K., Hussein, E. H., and Matthews, B. F. 2011. Posttranscriptional gene silencing of root-knot nematode in transformed soybean roots. Exp. Parasitol. 127:90-99.

Jones, J. T., Haegeman, A., Danchin, E. G. J., Gaur, H. S., Helder, J., Jones, M. G. K., Kikuchi, T., Manzanilla-López, R., Palomares-Rius, J. E., Wesemael, W. M. L., and Perry, R. N. 2013. Top 10 plant-parasitic nematodes in molecular plant pathology. Mol. Plant Pathol. 14:946-961.

Kim, S. H., and Hamada, T. 2005. Rapid and reliable method of extracting DNA and RNA from sweet potato, Ipomoea batatas (L). Lam. Biotechnol. Lett. 27:1841-1845.

Klink, V. P., Martins, V. E., Alkharouf, N. W., Overall, C. C., MacDonald, M. H., and Matthews, B. F. 2007. A decline in transcript abundance for Heterodera glycines homologs of Caenorhabditis elegans uncoordinated genes accompanies its sedentary parasitic phase. BMC Dev. Biol. 7:35. 
Li, J. R., Todd, T. C., Oakley, T. R., Lee, J., and Trick, H. N. 2010. Hostderived suppression of nematode reproductive and fitness genes decreases fecundity of Heterodera glycines Ichinohe. Planta 232:775-785.

Lin, M. S. 1989. Testing the resistance of sweet-potato varieties to potato-rot nematode by artificial inoculation. J. Nanjing Agric. Univ. 3:44-47.

Livak, K. J., and Schmittgen, T. D. 2001. Analysis of relative gene expression data using real-time quantitative PCR and the $2^{-\triangle \Delta C T}$ method. Methods 25: 402-408.

Nicol, J. M., Turner, S. J., Coyne, D. L., Den Nijs, L., Hockland, S., and Maafi, Z. T. 2011. Current nematode threats to world agriculture. Pages 21-43 in: Genomics and Molecular Genetics of Plant-Nematode Interactions. J. Jones, G. Gheysen, and C. Fenoll, eds. Springer, the Netherlands.

Oerke, E. C. 2006. Crop losses to pests. J. Agric. Sci. 144:31-43.

Overstreet, C. 2009. Nematodes. Pages 135-159 in: The Sweet Potato. G. Loebenstein and G. Thottappilly, eds. Springer, the Netherlands.

Schneider, C. A., Rasband, W. S., and Eliceiri, K. W. 2012. NIH Image to ImageJ: 25 years of image analysis. Nat. Methods 9:671-675.

Scurrah, M. I., Niere, B., and Bridge, J. 2005. Nematode parasites of Solanum and sweet potatoes. Pages 193-220 in: Plant Parasitic Nematodes in Subtropical and Tropical Agriculture. M. Luc, R. A. Sikora, and J. Bridge, eds. CABI Publishing, Wallingford UK.

Southey, J. F. 1986. Laboratory Methods for Work with Plant and Soil Nematodes. HMSO Books, London.

Souza Ddos, S., de Souza, J. D., Grossi-de-Sá, M., Rocha, T. L., Fragoso, R. R., Barbosa, A. E., de Oliveira, G. R., Nakasu, E. Y., de Sousa, B. A., and Pires, N. F. 2011. Ectopic expression of a Meloidogyne incognita dorsal gland protein in tobacco accelerates the formation of the nematode feeding site. Plant Sci. 180:276-282.

Subbotin, S. A., Madani, M., Krall, E., Sturhan, D., and Moens, M. 2005. Molecular diagnostics, taxonomy, and phylogeny of the stem nematode Ditylenchus dipsaci species complex based on the sequences of the internal transcribed spacer-rDNA. Phytopathology 95:1308-1315.

Urwin, P. E., Lilley, C. J., and Atkinson, H. J. 2002. Ingestion of doublestranded RNA by preparasitic juvenile cyst nematodes leads to RNA interference. Mol. Plant-Microbe Interact. 15:747-752.
Wan, F., Peng, D. L., Yang, Y. W., and He, Y. Q. 2008. Species specific molecular diagnosis of Ditylenchus destructor populations occurring in China. Acta Phytopathol. Sin. 38:263-270.

Wieczorek, K., Hofmann, J., Blöchl, A., Szakasits, D., Bohlmann, H., and Grundler, F. M. 2008. Arabidopsis endo-1,4- $\beta$-glucanases are involved in the formation of root syncytia induced by Heterodera schachtii. Plant J. 53: 336-351.

Xie, Y. P., Ma, D. F., Li, H. M., Li, Q., Wang, X., and Li, X. Y. 2010. Sweet potato stem-nematode expansibility resistance identification method. Sweet Potato Research Institute. Chinese Academy of Agricultural Sciences, China.

Xie, Y. P., Wang, X., Li, H. M., and Sun, H. J. 2009. Evaluation of sweet potato germplasm resources with double resistance to sweet potato rot nematode. J. Plant Genet. Resour. 10:136-139.

Xie, Y. Z., Yin, Q. H., Dai, Q. W., and Qiu, R. L. 2004. Inheritance and breeding for resistance to sweet potato nematodes. J. Plant Genet. Resour. 5:393-396.

Xu, Z., Zhao, Y. Q., Yang, D. J., Sun, H. J., Zhang, C. L., and Xie, Y. P. 2015. Attractant and repellent effects of sweet potato root exudates on the potato rot nematode, Ditylenchus destructor. Nematology 17:117-124.

Yadav, B. C., Veluthambi, K., and Subramaniam, K. 2006. Host-generated double stranded RNA induces RNAi in plant-parasitic nematodes and protects the host from infection. Mol. Biochem. Parasitol. 148:219-222.

Yang, J., Bi, H. P., Fan, W. J., Zhang, M., Wang, H. X., and Zhang, P. 2011. Efficient embryogenic suspension culturing and rapid transformation of a range of elite genotypes of sweet potato (Ipomoea batatas [L.] Lam.). Plant Sci. 181:701-711.

Zengel, J. M., and Epstein, H. F. 1980. Identification of genetic elements associated with muscle structure in the nematode Caenorhabditis elegans. Cell Motil. Cytoskeleton 1:73-97.

Zhang, S., Zhang, S., Wang, H., and Chen, Y. 2006. Characteristics of sweet potato stem nematode in China. Acta Phytopathol. Sin. 36:22-27.

Zhou, Z., Wang, X., Ma, D. F., Li, H. M., Xie, Y. P., and Li, X. Y. 2005. Identification of RAPD markers linked to stem-nematode resistant gene in sweet potato. J. Agric. Biotechnol. 13:549-552. 